\title{
Reversible Inhibitor Biosensor Systems in Dynamic Mode
}

\author{
Vania Rangelova*, Pavlina Kasarova and Vasil Spasov
}

Technical University Sofia, Branch Plovdiv, Bulgaria

\begin{abstract}
The biosensor amperometric transducers can work in the case of three basic types of reversible inhibitor enzyme systems - with competitive inhibition, with non-competitive inhibition and mixed inhibition. Typically they work in static mode. Now they are investigated in dynamic mode. The kinetic in those type biosensors is generally discussed in terms of a simple extension to the Michaelis-Menten reaction scheme. The investigated biosensors are amperometric product sensitive. The parameters for simulations are chosen from some real experiments with biosensors. The models are described in non-stationary diffusion conditions. Solving system of non-linear partial differential equations is received in three dimensional size and the concentration profiles in active membrane of substrate $S(x, t)$, inhibitor $I(x, t)$ and product $P(x, t)$ are received. The systems of non-linear differential partial equations have been solved numerically in MATLAB medium. The influence of starting concentration of substrate, inhibitor and kinetic parameters - reaction rate and reaction constants of biosensors over output current has been investigated.
\end{abstract}

Keywords: Amperometric biosensor; Inhibition; Dynamic mode; Simulations

\section{Notations}

$n=2$, Number of electrons taking part in the electrochemical reaction on the electrode surface

$$
\begin{aligned}
& \text { So- Starting concentration of the measured substrate } \\
& I o \text { - Starting concentration of the inhibitor } \\
& V_{s} \text { - Maximal reaction velocity, } \mathrm{mM} / \mathrm{s} \\
& d \text { - Thickness of active membrane, } \mu \mathrm{m} \\
& \text { F- Faraday's number, } \mathrm{F}=96,5 \mathrm{~A} . \mathrm{s} / \mathrm{mmol}
\end{aligned}
$$

$A$ - Area of the cathode of the indicator electrode, $\mathrm{m}^{2}$

$K s$ - Reaction constant for substrate $S, \mathrm{mM}$

$K_{I}$ - Reaction constant for inhibitor toward $E, \mathrm{mM}$

$K_{I^{\prime} \text { - }}$ Reaction constant for inhibitor toward ES, mM

$K p$ - Reaction constant for product, $\mathrm{mM}$

Ds- Diffusion coefficient of substrate, $\mathrm{m}^{2} / \mathrm{s}$

$D_{I}$ - Diffusion coefficient of inhibitor, $\mathrm{m}^{2} / \mathrm{s}$

Dp-Diffusion coefficient of product, $\mathrm{m}^{2} / \mathrm{s}$

\section{Introduction}

The biosensors now days are very popular devices and have a very big area of uses from health-care industry through food quality appraisal to environmental monitoring. It is due to their main advantages-to detect concentration in very low range of different substances cheap, selective and highly sensitive. Measurements in enzyme based biosensor systems with enzyme layers are either made through stationary mode or flow-through mode [1]. Our investigation is done over stationary mode biosensors. Now days are used different sensing principles for determining the concentration of substrateelectrochemical, calorimetric, optical and piezoelectric. Biosensor systems with enzyme inhibition are very popular in environmental monitoring [1] used with a proper intelligent terminals [2] they could do their job - to serve humanity for better life. Recently biosensors are used for the detection of pollutants, such as pesticides, heavy metal ions and other toxic compounds. Botre et al. [3] use inhibition-based biosensor for the detection of environmental contaminants. A new bienzymatic inhibition biosensor based on the combined catalytic activity of the enzymes alkaline phosphatase and glucose oxidase is proposed and discussed for the direct determination of 2,4-dichlorophenoxyacetic acid (2,4-D), one of the most powerful and diffused defoliants, which also is endowed with estrogenic properties. Lee $S$ et al. [4] use ureaseimmobilized biosensor for the determination of heavy-metal ions. Multinzime biosensors systems are investigated also from Ganesan SP et al. [5], but again is investigated only the same steady-state mode. Generally those types of biosensors - stationary mode, work in steady state mode and the majority research papers are in this mode and dynamic mode is still poorly examined. But it is known that the decay time of transient process is long (for tissue biosensors 3 to $5 \mathrm{~min}$ ) and therefore dynamic measurements can be used. A comprehensive study of the mathematical modeling of amperometric biosensors is given in [6]. Recently Baronas et al. are developed a mathematical models of amperometric product-sensitive biosensors in dynamic mode [7-9]. Baronas et al. [7] have a lots of papers dedicated especially on modeling biosensors, so they wrote a book called - "Mathematical modeling of biosensors". Where are done very comprehensive mathematical modeling and simulations on biosensors [10]. In the chapter 8 very shortly they investigate the enzyme inhibitions, they describe and gave solutions for substrate and product inhibition using Michaele- Menteh kinetic but their equations don't include the inhibitor equation. They use only substrate inhibition constants - Ks which is not enough to describe the full model of the enzymatic reaction. In our paper are investigated three type of inhibition. Yupeng Liu [11] had done some interesting modeling and simulation and dynamic mode also for free enzyme model electrochemical biosensor, but it is not inhibitor type biosensor.

${ }^{*}$ Corresponding author: Vania Rangelova, Technical University Sofia, Branch Plovdiv, Bulgaria, E-mail: vaniarangelova@tu-plovdiv.bg

Received October 01, 2018; Accepted October 31, 2018; Published November 11, 2018

Citation: Rangelova V, Kasarova P, Spaso V (2018) Reversible Inhibitor Biosensor Systems in Dynamic Mode. J Biosens Bioelectron 9: 260. doi: 10.4172/21556210.1000260

Copyright: (c) 2018 Rangelova V, et al. This is an open-access article distributed under the terms of the Creative Commons Attribution License, which permits unrestricted use, distribution, and reproduction in any medium, provided the original author and source are credited. 
The goal of this paper is to investigate the all three different type of enzyme inhibitor kinetics models of biosensors in dynamic mode, and they are - with competitive inhibition, with non-competitive inhibition and mixed inhibition. In the enzyme reactions, enzyme $E$ and substrate $S$ react and ES complex is formed. After that $E S$ is transformed to a transition complex $E S$. The latter convert $S$ to product $P$ and the result is free enzyme and product

$$
E+S<-\longrightarrow E S<-\longrightarrow E S^{*}<-\longrightarrow E P<-\longrightarrow E+P
$$

The kinetic of those reaction is $v=\frac{V_{S} S}{K_{S}+S}$ and connect enzyme reaction rate $\mathrm{v}$ with concentration of substrate $S$ and two constants, maximal reaction velocity $V_{s}$ and Michaelis-Menten constant for given substrate $K_{S}$. Those two are called kinetic parameters.

\section{Presentation of Mathematical Models}

Enzyme inhibitors are molecules that bind to enzymes and decrease their activity [12]. Loss of activity may be either reversible, where activity may be restored by the removal of the inhibitor, or irreversible, where the loss of activity is time dependent and cannot be recovered during the timescale of interest. The main is that kinetic parameters $K_{s}$ and $V_{s}$ are changed. More important for most enzyme-catalyzed processes is the effect of reversible inhibitors. These are generally discussed in terms of a simple extension to the Michaelis-Menten reaction scheme.

There is known three type reversible inhibition: Competitive inhibitions, Non-competitive inhibitions (sub type - Uncompetitive inhibitions, rarely encountered and we don't investigate it) and Mixedtype inhibitions.

Competitive inhibitors can bind to $E$, but not to ES. Competitive inhibition increases $K_{s}$ (i.e., the inhibitor interferes with substrate binding), but does not affect $V_{S}$ (the inhibitor does not hamper catalysis in $E S$ because it cannot bind to $E S$ ).

Non-competitive inhibitors have identical affinities for $E$ and $E S$ $\left(K_{\mathrm{I}}=K_{\mathrm{I}}^{\prime}\right)$. Non-competitive inhibition does not change $K_{\mathrm{S}}$ (i.e., it does not affect substrate binding) but decreases $V_{S}$ (i.e., inhibitor binding hampers catalysis).

Uncompetitive inhibitors bind only to ES complexes at locations other than the catalytic site. Substrate binding modifies enzyme structure, making inhibitor- binding site available. Inhibition cannot be reversed by substrate. Apparent $V_{S}$ decreased; $K_{S}$ is decreased.

Mixed-type inhibitors bind to both $E$ and $E S$, but their affinities for these two forms of the enzyme are different $\left(K_{I} \neq K_{I}\right)$. Thus, mixed-type inhibitors interfere with substrate binding (increase $K_{\mathrm{s}}$ ) and hamper catalysis in the ES complex (decrease $V_{S}$ ).

The reaction follows the schemes, given in the Figure 1.

Where $I$ represents the reversible inhibitor and the inhibitory (dissociation) constants $K_{I}$ and $K_{I}^{\prime}, K_{\text {cat }}$ is the rate constant of product formation.

We will investigate biosensors systems only in active membrane, because it is known that concentration of substrate $S(x)$ and inhibitor $I(x)$ in other two membranes are changed linear. Biosensors are operated under diffusion control. We admit that electrode has symmetrical geometry and enzyme is homogeneous distributed in active membrane. We assume that diffusion is one-dimensional in space and is described with second Fick's law. The equation described those amperometric systems in dynamic mode is

$$
\frac{\partial Q}{\partial t}=D_{Q} \frac{\partial^{2} Q}{\partial x^{2}}+R_{Q}
$$

Where $\mathrm{Q}$ is the concentration of any species involved in enzyme or electrochemical reactions, $D_{Q}$ is the corresponding diffusion constant for the active membrane and $R_{Q}$ is the term related with enzyme kinetic.

For the competitive reversible inhibition systems $R_{Q}$ is

$$
R_{Q 1}=\frac{V_{S}}{K_{S}\left(1+\frac{I}{K_{I}}\right)+S} S
$$

For non - competitive reversible inhibition system $R_{Q}$ is

$$
R_{Q_{2}}=\frac{V_{S}}{\left(1+\frac{I}{K_{I}}\right)} \frac{S}{\left(K_{S}+S\right)}
$$

And for mixed reversible inhibition system $\mathrm{R}_{\mathrm{Q}}$ is

$$
R_{Q_{3}}=\frac{V_{S} \cdot S}{K_{S}\left(1+\frac{I}{K_{I}}\right)+S\left(1+\frac{I}{K_{I}^{\prime}}\right)},
$$

Where: Ks-reaction rate constant for substrate; $K_{I}$ and $K_{I}{ }^{\prime}$ - for inhibitor; $\mathrm{V}_{\mathrm{s}}$ - maximal velocity of enzyme reaction - enzymatic rate.

Taking in mind all that the dynamic mode for the three type of biosensors will be described with the following system of partial differential equations -

For the competitive inhibition

$$
\begin{aligned}
& \frac{\partial S}{\partial t}=B \frac{\partial^{2} S}{\partial x^{2}}-R_{Q 1} \\
& \frac{\partial I}{\partial t}=D_{I} \frac{\partial^{2} I}{\partial x^{2}}-R_{Q 1} \\
& \frac{\partial P}{\partial t}=D \frac{\partial^{2} P}{\partial x^{2}}+R_{Q 1}
\end{aligned}
$$
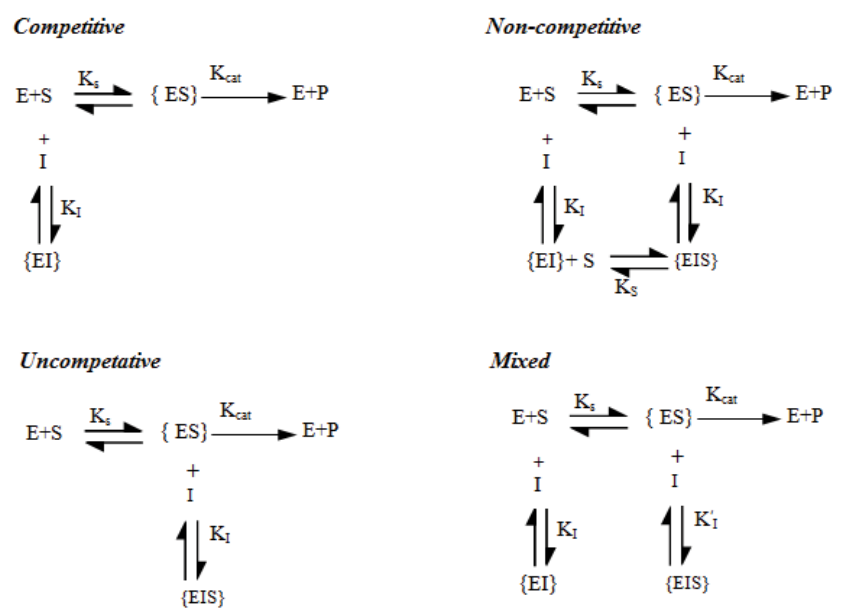

Source: Authors

Figure 1: The reaction schemes of three type inhibitions. 
for non - competitive reversible inhibition

$$
\begin{array}{ll}
\frac{\partial S}{\partial t}=B \frac{\partial^{2} S}{\partial x^{2}}-R_{Q 2} & \frac{\partial I}{\partial t}=D_{I} \frac{\partial^{2} I}{\partial x^{2}}-R_{Q 2} \\
\frac{\partial P}{\partial t}=p \frac{\partial^{2} P}{\partial x^{2}}+R_{Q 2} &
\end{array}
$$

for mixed reversible inhibition

$$
\begin{gathered}
\frac{\partial S}{\partial t}=D \frac{\partial^{2} S}{\partial x^{2}}-R_{Q 3} \quad \frac{\partial I}{\partial t}=D_{I} \frac{\partial^{2} I}{\partial x^{2}}-R_{Q 3} \\
\frac{\partial P}{\partial t}=D \frac{\partial^{2} P}{\partial x^{2}}+R_{Q 3}
\end{gathered}
$$

The output current is proportional to gradient of the product concentration at the electrode surface

$$
I=\left.n F A D_{P} \frac{\partial P}{\partial x}\right|_{x=d},[\mathrm{~A}]
$$

Let we denote $x=0$ for the bulk/membrane interface and $x=d$ for the electrode surface. The action in biosensor starts when some quality of substrate is appears into biological recognition element - active membrane. The initial conditions are

$$
t=0 \quad S(x, 0)=\text { So } \quad I(x, 0)=\operatorname{Io} \quad P(x, 0)=0
$$

Limiting conditions are

$$
x=0 \quad S(0, t)=\text { So } \quad I(0, t)=I o \quad P(0, t)=0
$$

The substrate, and inhibitor didn't react with the electrode, but product fully exhausted. The medium is well stirred, then the limiting conditions are

$$
\begin{aligned}
& x=d \\
& \left.\frac{\partial S}{\partial x}\right|_{x=d}=0,\left.\quad \frac{\partial I}{\partial x}\right|_{x=d}=0 P(d, t)=0 .
\end{aligned}
$$

\section{Results from Digital Simulations and Discussion}

For solving systems of non-linear partial differential equations (PDE) we use Matlab solver pdepe [5-7]. It use both finite difference and finite element methods as described in [13]. Pdepe solve initialboundary value problems for system of parabolic-elliptic PDEs in the one space variable $x$ and time $t$. The ordinary differential equations resulting from discretization in space are integrated to obtain approximate solutions at times specified in a time vector. Time vector specifying the points at which a solution is requested for every value in distance vector. The pdepe function returns values of the solution on a mesh provided in a distance vector. Distance vector specifying the points at which a numerical solution is requested for every value in time vector. Three programs defining the systems are developed, some appropriate substitutions were made, and also canonization of the equations, as well as fixation of the physical parameters.

And so will be investigated amperometric product sensitive inhibitor biosensor system. The parameters are chosen from some real experiments with biosensors from the authors [14-17] and for the simulations they are

$$
n=2, \text { So }=5 \mathrm{mM}, I o=0,5 \mathrm{mM}, V_{S}=0,5 \mathrm{mM} / \mathrm{s}-\text { or changed }
$$

$F=96,5$ A.s $/ \mathrm{mmol}$ - Faraday's number, $A=7,85.10^{-7} \mathrm{~m}^{2}$ diameter of cathode is $1 \mathrm{~mm}$

$$
\begin{aligned}
& K s=0.6 \mathrm{mM}-\text { reaction constant for substrate, } \\
& K_{I}=0.6 \mathrm{mM}-\text { reaction constant for inhibitor, }
\end{aligned}
$$

$$
\begin{aligned}
& K_{I^{\prime}}=2 \mathrm{mM}-\text { reaction constant for inhibitor, } \\
& K p=2 \mathrm{mM}-\text { reaction constant for product, - or changed, } d=70 \mu \mathrm{m}, \\
& D s=1,5.10^{-9} \mathrm{~m}^{2} / \mathrm{s}, D_{I}=5,5.10^{-9} \mathrm{~m}^{2} / \mathrm{s}, D p=2,5.10^{-9} \mathrm{~m}^{2} / \mathrm{s} .
\end{aligned}
$$

Concentration profiles of substrate $S(x, t)$, inhibitor $I(x, t)$ and product $P(x, t)$, for the biosensor with competitive inhibitions

In Figure 2, in three dimensional coordinates are given solutions for the concentration profiles of substrate $S(x, t)$, inhibitor $I(x, t)$ and product $P(x, t)$, measurement unit is in $\mathrm{mM}$, in the active membrane of the biosensor with selected thickness equal to $d=70 \mu \mathrm{m}$. Time is taken to be $t=5 \mathrm{~s}$ and reaction rate $V_{s}=0,5 \mathrm{mM} / \mathrm{s}$. The value of starting concentration of substrate is chosen to be $5 \mathrm{mM}$ which range is normal for the electrochemical biosensors. Reaction constants for substrate and inhibitor are chosen equal. In the up part of the Figure 2 are results for the case without inhibitor, so they are for concentration of $\mathrm{I}=0 \mathrm{mM}$. The biosensor react like ordinary biosensor, the output product reaches maximal concentration of $0,12 \mathrm{mM}$, after that is fully exhausted at the electrode. Product is equal to zero at the beginning of the membrane and time, because still is not formed. Because the system is product sensitive the product concentration is increasing with the consuming of the substrate. The lower part of the Figure 1 shows the profiles when there has presence of inhibitor, for $\mathrm{I}=5 \mathrm{mM}$. It is seen that inhibitor is consumed very rapidly. The reaction constants for substrate and inhibitor are chosen equal, so due to the competition the output product reaches very small concentration of $0.08 \mathrm{mM}$, it is 1.5 times lower then when has no inhibitor in the system.

\section{Concentration profiles of substrate $S(x, t)$, inhibitor $I(x, t)$ and product $P(x, t)$, for the biosensor with with non-competitive inhibitions}

In Figure 3 are given the same reagents $S(x, t), I(x, t)$ and $P(x, t)$ but for the biosensor with non-competitive inhibitions. The starting conditions are equal, so the resulting profiles have no big difference. Here the output product reaches maximal concentration of $0,025 \mathrm{mM}$ which is 4,8 times smaller than when has no inhibition the reason is, the decreasing of the maximal reaction rate $V_{S}$ in the presence of inhibitor.

\section{Concentration profiles of substrate $S(x, t)$, inhibitor $I(x, t)$ and product $P(x, t)$, for the biosensor with mixed inhibitions}

In Figure 4 are given the same reagents $S(x, t), I(x, t)$ and $P(x, t)$ but for the biosensor with mixed inhibitions. Now 2 reactions are in action. The inhibitor bind to both $E$ and $E S$, so we use two different reaction constants and their affinities for these two forms of the enzyme are different, for the enzyme $E, K_{I}=0,6 \mathrm{mM}$ - reaction constant for inhibitor, and for the complex ES, we chose 3 times bigger constant $K_{I^{\prime}}=2 m M$ - reaction constant for inhibitor. Other parameters are the same - reaction constant for substrate, all corresponding diffusion constants, starting concentration of substrate. Here the output product reaches maximal concentration of $0,004 \mathrm{mM}$ which is 30 times smaller than when has no inhibition. The reason is the decreasing of the maximal reaction rate $V_{S}$ in the presence of inhibitor and it influents over the both-enzyme activity and enzyme substrate complex activity. And the concentration of the inhibitor stays in very big values- almost the same, in previous two types of inhibition the inhibitor almost fully exhausted.

Now we will investigate the influence of starting concentration So, $I o$, kinetic parameters - Vs, Ks and reaction constant of inhibitor $K_{I}$ over output current of biosensors. 

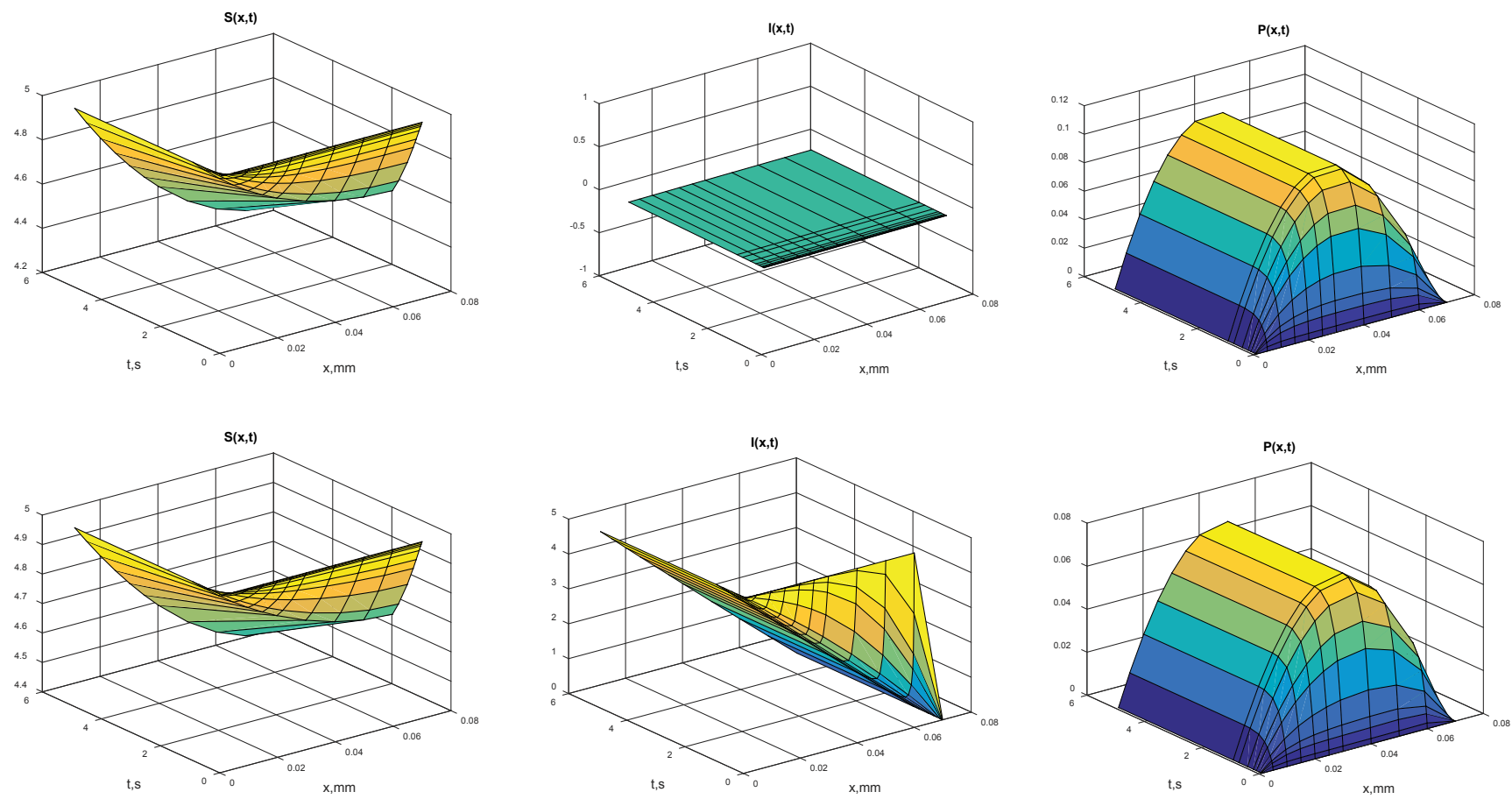

Source: Authors

Figure 2: Competitive inhibition.
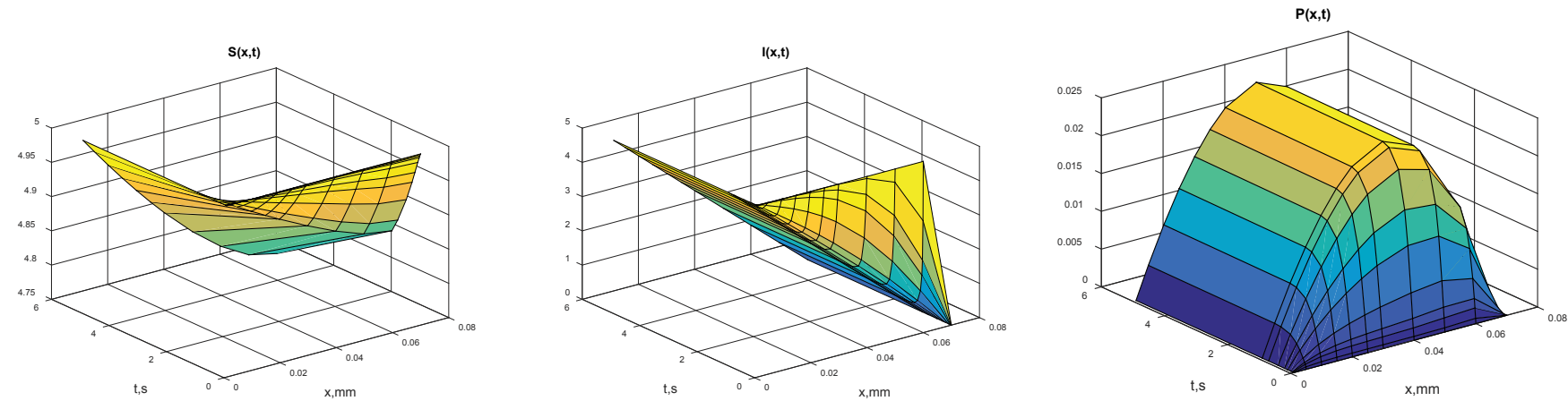

Source: Authors

Figure 3: Non - Competitive inhibition
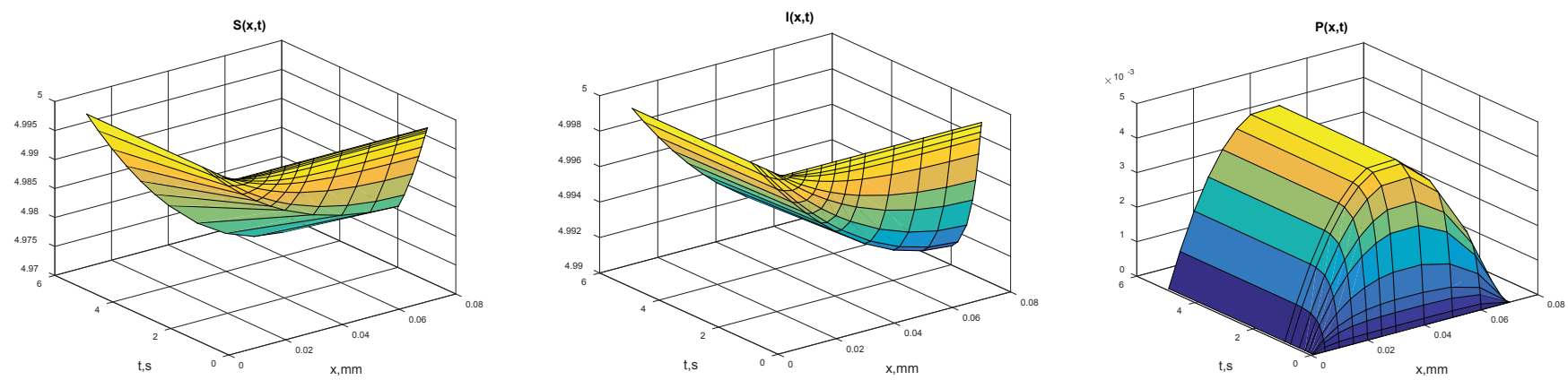

Source: Authors

Figure 4: Mixed inhibition. 
Citation: Rangelova V, Kasarova P, Spaso V (2018) Reversible Inhibitor Biosensor Systems in Dynamic Mode. J Biosens Bioelectron 9: 260. doi: $10.4172 / 2155-6210.1000260$

Page 5 of 9

Influence of starting concentration So, Io, kinetic parameters - Vs, Ks and reaction constant of inhibitor $K_{I}$ over output current of biosensors for the biosensor with competitive inhibition

The influence of starting concentration So of measured substrate over the output current $I$ of the biosensor with competitive inhibition is given to the Figure $5 \mathrm{a}$. We gave the calculated relation of $I o$ to So. The concentration of $I=2 \mathrm{mM}$. For the relation $I o / S o=0,001$, So is 1000 times bigger than $I o$. With increasing of So from $2 \mathrm{mM}$ to $2000 \mathrm{mM}$ the output current is increase too, which is obviously from the kinetics reaction, more substrate - bigger output current. For the So from $2 m M$, current is $I=34 n A$ and for the So from $2000 m M$, current is $I$ $=58 \mathrm{nA}$. And after $20 \mathrm{mM}$ the biosensor system is fully saturated so no significant changes in the output are possible. This kind of dependence, which distinguishes the enzymatic reactions from the other chemical processes, is due to the intermediate formation of enzyme-substrate complex (ES) during the enzyme-catalyzed reaction, and when substrate is in very big concentration - it reaches saturation of all active substrate centers.
The influence of starting concentration of $I o$ is given to the Figure $5 \mathrm{~b}$. For the two values of inhibitor, $I=0 \mathrm{mM}$ and $I=5 \mathrm{mM}$ the output current decrease from $54 n \mathrm{~A}$ to $40 \mathrm{nA}$, due to the presence of

Inhibitor. Naturally those results depend significally and from values of the other reagents, but in common the result is decreasing the output current.

The influence of kinetic parameters - maximal reaction velocity $V s$ is given to the Figure 5c. Again in relation, so for the $S o / V s=50$, So is 50 times bigger than $V s$. With increasing the reaction velocity $V s$ from $0.1 \mathrm{mM} / \mathrm{s}$ to $5 \mathrm{mM} / \mathrm{s}$ the output current is increasing too, because the enzyme reaction velocity is proportional to maximal reaction velocity. For the values of $5 \mathrm{mM} / \mathrm{s}$ is seen the appearance of maximal response like in a second order oscillating unit with a decay factor of less than 1 . It is only for the transient time, until is reached the steady state line. So the biosensor system looks like an oscillating unit with a decay factor.

The influence of kinetic parameters - reaction constant for substrate $K s$ is given to the Figure 5d. a
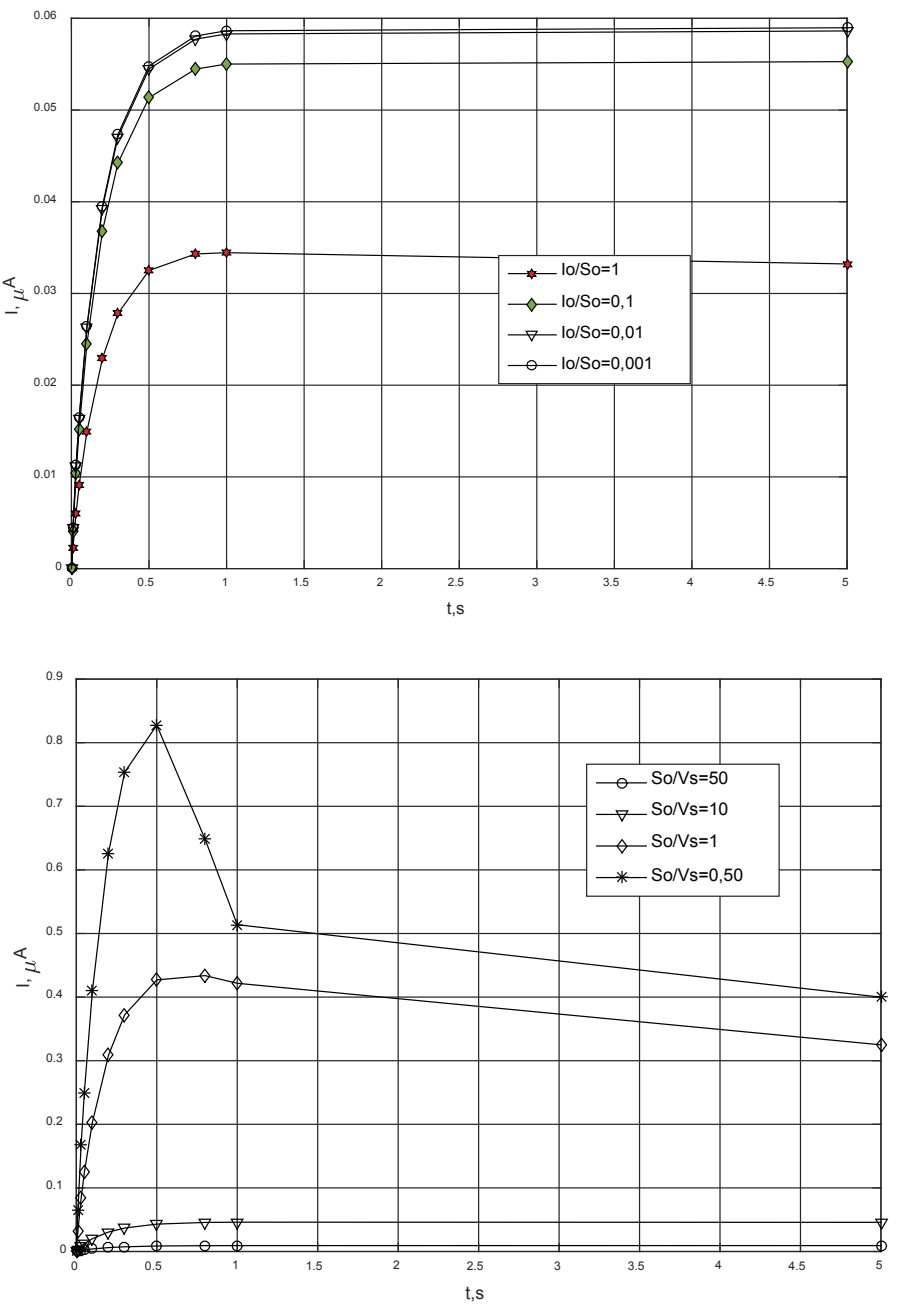

$\mathrm{b}$
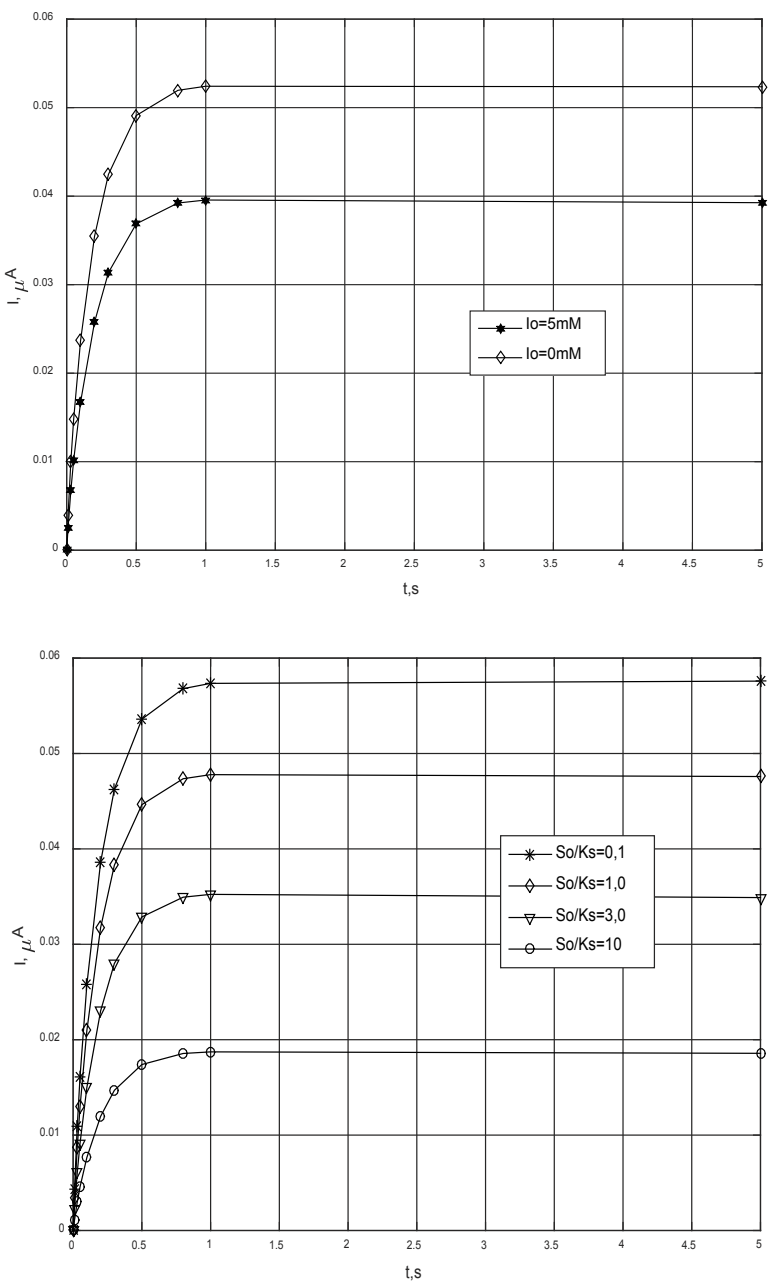

d

a) Influence of So, b) Influence of lo, c) Influence of Vs., d) Influence of Ks. 
Citation: Rangelova V, Kasarova P, Spaso V (2018) Reversible Inhibitor Biosensor Systems in Dynamic Mode. J Biosens Bioelectron 9: 260. doi: $10.4172 / 2155-6210.1000260$

Page 6 of 9

The bigger value of maximal reaction velocity $V s$ leads to bigger output current, the bigger value of Michaelis-Menten constant for given substrate $K s$ leads to smaller output current. It is because we sad in the beginning that the enzyme kinetic has the reaction velocity of the kind

$$
v=\frac{V_{S} S}{K_{S}+S}
$$

Influence of starting concentration So, Io, kinetic parameters - Vs, Ks and reaction constant of inhibitor $K_{I}$ over output current of biosensors for the biosensor with non - competitive inhibition

The influence of starting concentration So of measured substrate over the output current $I$ of the biosensor with non-competitive inhibition is given to the Figure 6a. We use the same values of all parameters only the equations are different. And form graph is seen that output current increases but in much values, due to the inhibition of the both complexes - enzyme and enzyme-substrate. For the So from

a
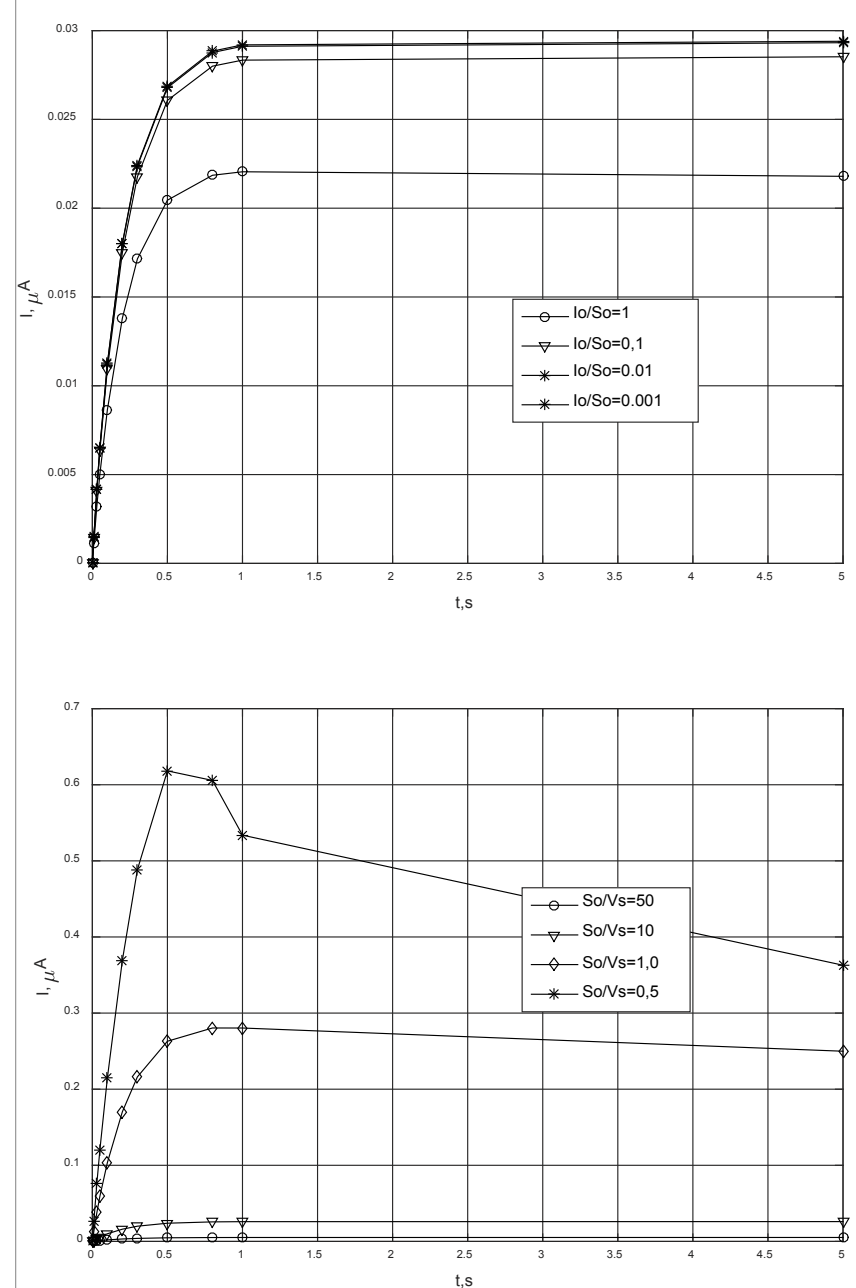

c
$2 \mathrm{mM}$, current is $I=24 \mathrm{nA}$ and for the So from $2000 \mathrm{mM}$, current is $I$ $=28 n A$. Here the max deviation is only $16.6 \%$, but for the competitive inhibition it was $70 \%$.

The influence of starting concentration of $I o$ is given to the Figure $6 \mathrm{~b}$. For the three values of inhibitor concentration, $I=0 \mathrm{mM}, I=3 \mathrm{mM}$ and $I=5 \mathrm{mM}$ the output current decrease from $62 n \mathrm{~A}$ to $23 \mathrm{nA}$, to $18 \mathrm{nA}$, due to the presence of inhibitor. Here for the starting concentration of the inhibitor $I=0 m M$, the output current is slightly bigger - $62 n A$, but for the presence of the inhibitor $I=5 \mathrm{mM}$ where we do the research it values is lower $-18 n A$ versus $39 n A$ for non-competitive inhibition.

The influence of kinetic parameters- maximal reaction velocity $V s$ is given to the Figure 6c. Again in relation, so for the $S o / V s=50$, So is 50 times bigger than $V s$. With increasing the reaction velocity $V s$ from $0.1 \mathrm{mM} / \mathrm{s}$ to $5 \mathrm{mM} / \mathrm{s}$ the output current is increasing too, because the enzyme reaction velocity is proportional to maximal reaction velocity. For the values of $5 \mathrm{mM} / \mathrm{s}$ is seen the appearance of maximal response. Its value is $620 \mathrm{nA}$, for the competitive inhibition it is $820 \mathrm{nA}$, and it is obvious from this enzymatic type of action of inhibitor. b
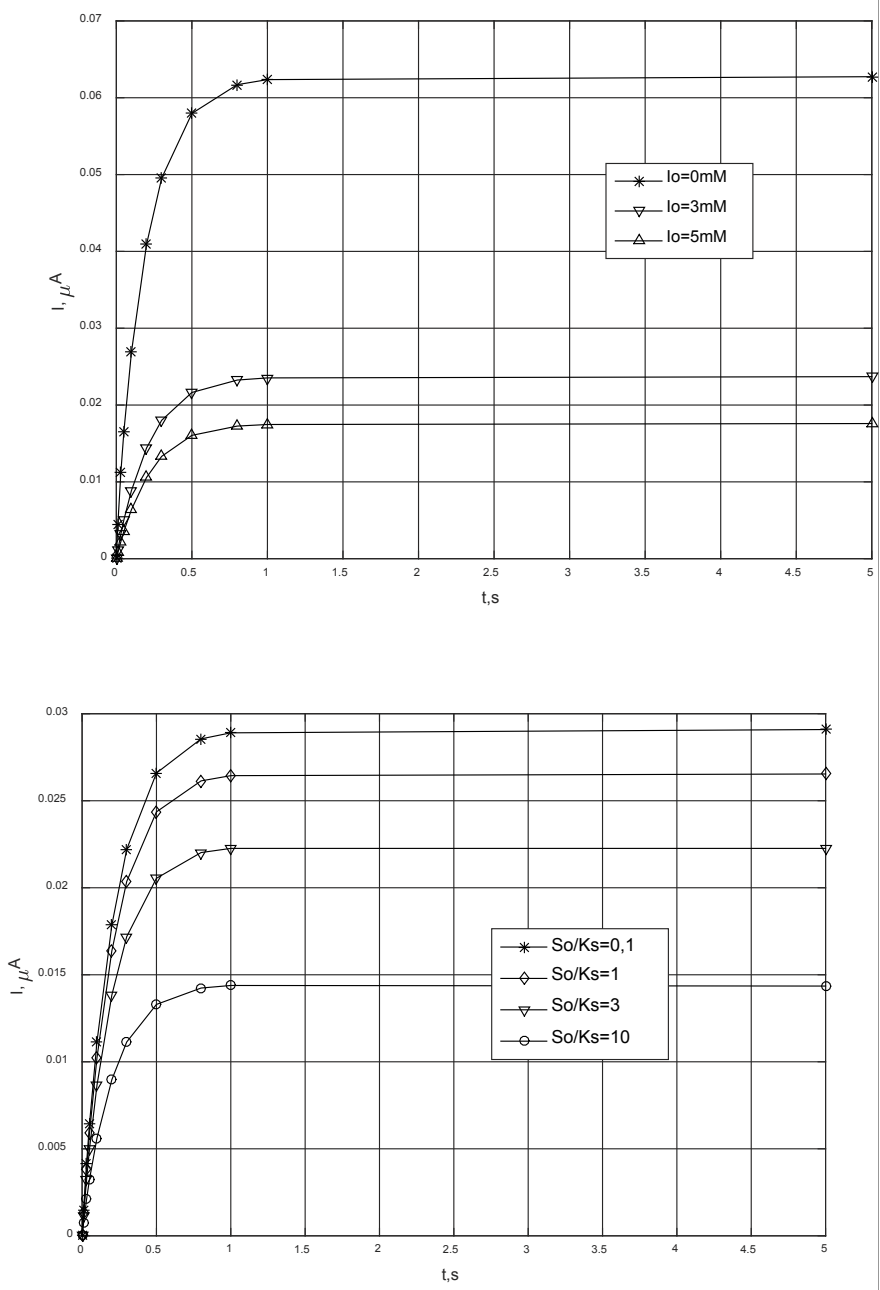

d

Figure 6: Non-Competitive inhibition

a) Influence of So, b) Influence of lo, c) Influence of Vs, d) Influence of Ks. 
Citation: Rangelova V, Kasarova P, Spaso V (2018) Reversible Inhibitor Biosensor Systems in Dynamic Mode. J Biosens Bioelectron 9: 260. doi: $10.4172 / 2155-6210.1000260$

Page 7 of 9

The influence of kinetic parameters - reaction constant for substrate $K s$ is given to the Figure $6 \mathrm{~d}$.

The bigger value of maximal reaction velocity $V s$ leads to bigger output current, the bigger value of Michaelis-Menten constant for given substrate $K s$ leads to smaller output current.

Influence of starting concentration So, Io, kinetic parameters - Vs, Ks and reaction constant of inhibitor $K_{I}$ over output current of biosensors for the biosensor with mixed inhibition

The influence of starting concentration So of measured substrate over the output current $I$ of the biosensor with mixed inhibition is given to the Figure $7 \mathrm{a}$. What is seen form the graph here - for increasing of measured substrate So from $2 m M$ to $2000 \mathrm{mM}$, the output current has not any deviation, or it is so small that will not measure at all. Or will be with the range of measurement error 3-4 $n A$.

The influence of starting concentration of $I o$ is given to the Figure $7 \mathrm{~b}$. Here the same subordination continues, output values of the current began smaller and smaller, and smaller than previous two types of kinetics. They now are several $n A$, from 2 to $9 n A$.

The influence of kinetic parameters - maximal reaction velocity

a
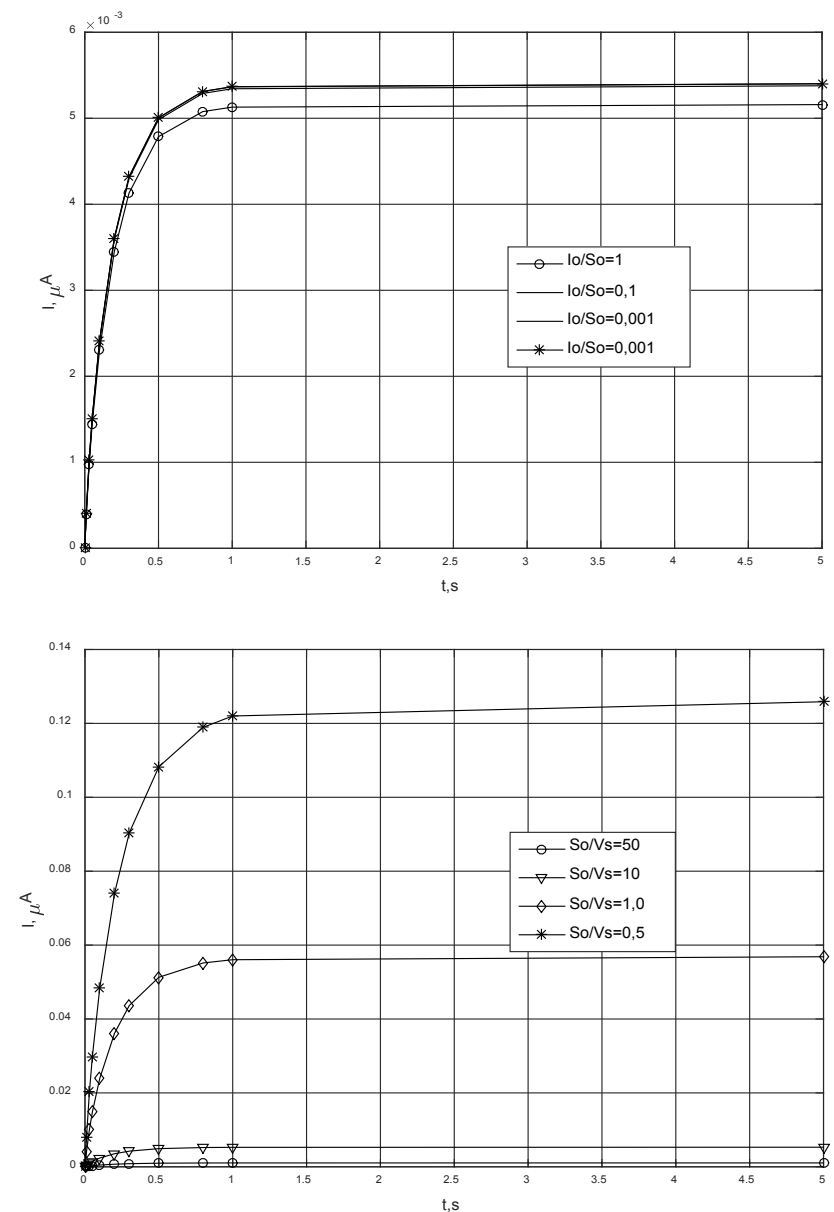

c
$V s$ is given to the Figure 7c. With increasing the reaction velocity $V s$ from $0.1 \mathrm{mM} / \mathrm{s}$ to $5 \mathrm{mM} / \mathrm{s}$ the output current is increasing too, because the enzyme reaction velocity is proportional to maximal reaction velocity, but now due to the influence of the both complexes, there has no maximal response like in a second order oscillating unit. And the transient process for the biosensor is like in a first order oscillating unit.

The influence of kinetic parameters - reaction constant for substrate $K s$ is given to the Figure $7 \mathrm{~d}$.

The bigger value of maximal reaction velocity $V s$ leads to bigger output current, the bigger value of Michaelis-Menten constant for given substrate $K s$ leads to smaller output current. Again the output current is in very small values - 4-5 $n A$.

In the Figure 8 is given the output current $I$ in $u A$ in one graph - for biosensor with competitive inhibitions - the upper 3 lines, for the biosensor with non-competitive inhibitions - the middle three lines, and for the biosensor with mixed inhibitions - the down three lines.

\section{Conclusion}

Generally described electrochemical amperometric biosensors work in steady state mode. In this paper has done an attempt to

b
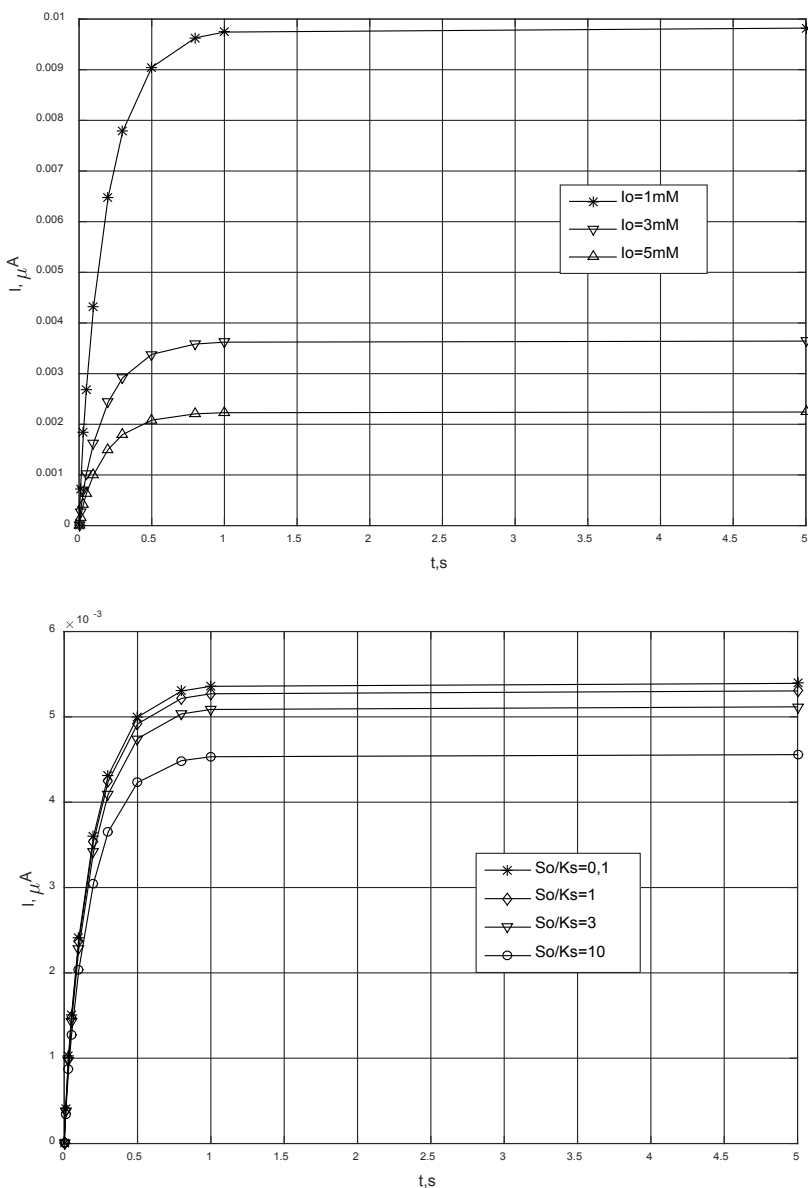

d

Figure 7: Mixed inhibition a) Influence of So, b) Influence of lo, c) Influence of Vs, d) Influence of Ks. 
Citation: Rangelova V, Kasarova P, Spaso V (2018) Reversible Inhibitor Biosensor Systems in Dynamic Mode. J Biosens Bioelectron 9: 260. doi: $10.4172 / 2155-6210.1000260$

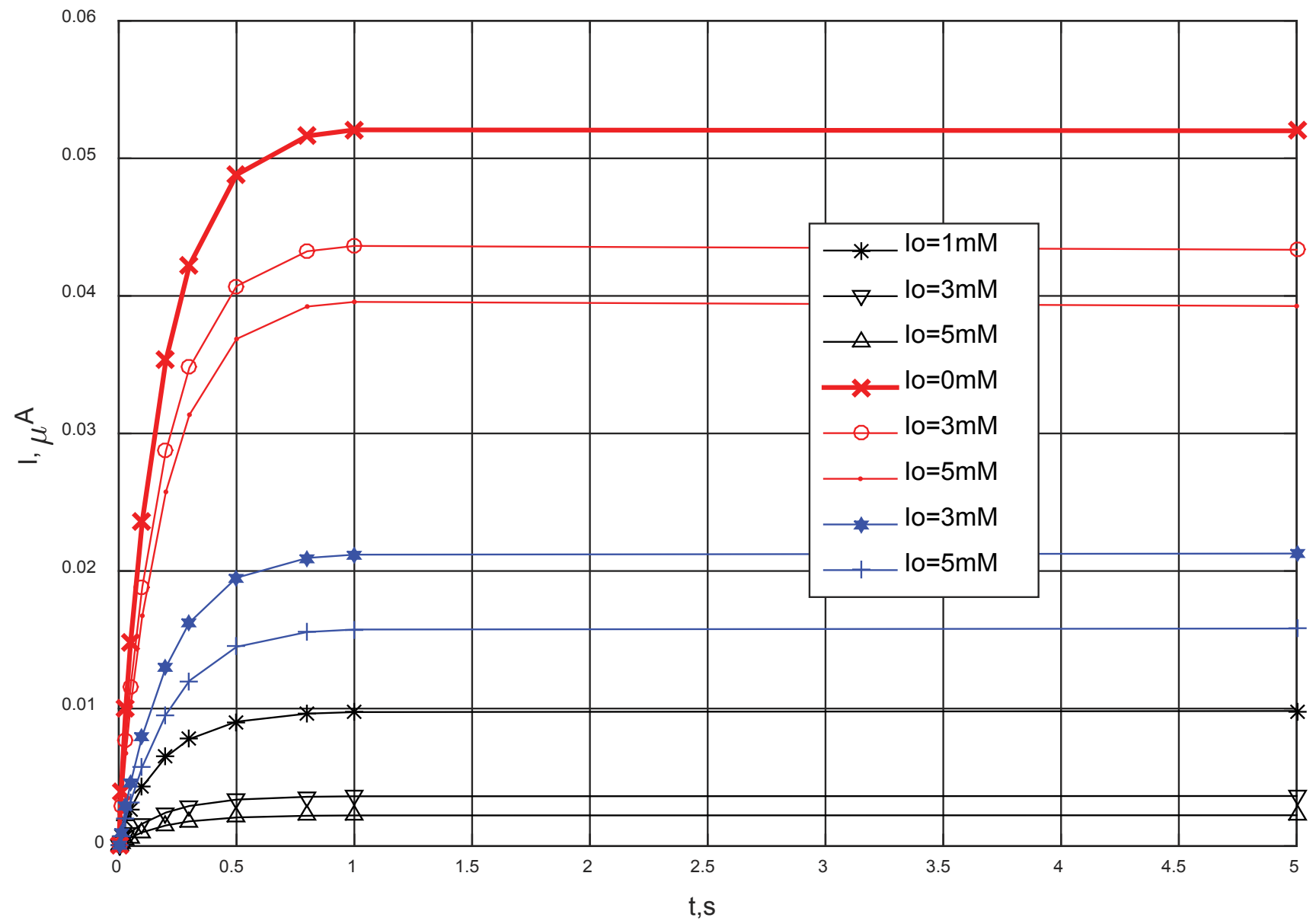

Figure 8: The output current I in uA for biosensor with competitive inhibitions - the upper 3 lines, biosensor with non-competitive inhibitions - the middle three lines, and biosensor with mixed inhibitions - the down three lines.

investigate the dynamic mode of the specific type amperometric biosensors - those with reversible inhibition. Mathematical modeling of the acting equations were performed and solution of those in the MATLAB environment. The results show that simulations are adequate; the concentration profiles of the three reagents can be seen. In addition are done investigation of the influence of starting concentration of substrate measured $S o$, and inhibitor $I o$, kinetic parameters- $V s, K s$ and reaction constant of inhibitor $K_{I}$ over output current of biosensors. For the further could be investigatre the influence of diffusion parameters Diffusion coefficient of substrate, Diffusion coefficient of inhibitor and Diffusion coefficient of product.

\section{References}

1. Kuswandi B, Mascini M (2005) Enzyme Inhibition Based Biosensors for Environmental Monitoring Current Enzyme Inhibition 1: 207-221.

2. Elenkov A (1997) Peripheral devices in computing systems.

3. Botrè $C$, Botrè $F$, Mazzei $F$, Podestà $E$ (2000) Inhibition-based biosensors for the detection of environmental contaminants. Environmental Toxicology and Chemistry 19: 2876-2881.

4. Lee S, Lee WY (2002) Determination of Heavy Metal lons Using Conductometric Biosensor Based on Sol-Gel-Immobilized Urease. Bull Korean Chem Soc 29: 1169

5. Ganesan SP, Saravanakumar K, Rajendran L (2014) Mathematical Modeling of Multienzyme Biosensor. Inter J Computational Mathematics.
6. Schulmeister T (1990) Mathematical modelling of the dynamic behaviour of amperometric enzyme electrodes. Selective Eletrode Rev 12: 203-260.

7. Baronas R, Ivanauskas F, Kulys J (2003) The Influence of the Enzyme membrane Thickness on the Response of Amperometric biosensors. Sensors 3: 248-262.

8. Baronas R, Ivanauskas F, Kulys J (2003) Computer Simulation of the Response of Amperometric biosensor in Stirred and non Stirred Solution. Nonlinear Analysis: Modelling and Control 8: 3-18.

9. Baronas R, Kulys J (2006) Modelling of Amperometric Biosensors in the Case of Substrate Inhibition. Sensors 6: 1513-1522.

10. Baronas R, Romas, Ivanauskas F, Feliksas, Kulys J, et al. Mathematica Modeling of Biosensors.

11. Liu Y, Qi W (2008) Mathematical and computational modeling of biosensors: Modeling for enzyme-substrate interaction and biomolecular interaction. Topics on Computational Biology and Chemistry.

12. Chaplin M, Bucke C (1990) Enzyme Technology, Cambridge University Press.

13. Skeel RD, Berzins M (1990) A method for the spatial discretization of parabolic equations in one space variable. J Scient Stat Comput 11: 1-32.

14. Rangelova V, Neykov A, Raikinska I (2002) Identification of a real tissue biosensor transfer function Application of Mathematics and Engineering and Economics'27. Heron Press, Sofia.

15. Rangelova V (2003) Influence of velocity of enzyme responses of tissue biosensors in the presence of substrate measurements in tissue. Scientific Works of the Union of Scientists in Bulgaria-Plovdiv, series B. Technics and Technologies 13: 232-236. 
Citation: Rangelova V, Kasarova P, Spaso V (2018) Reversible Inhibitor Biosensor Systems in Dynamic Mode. J Biosens Bioelectron 9: 260. doi: 10.4172/2155-6210.1000260

Page 9 of 9

16. Neykov Al, Kacarova P, Rangelova V (2003) Method for the diagnosis of biosensor with two dialysis membranes under limiting diffusion conditions 1 : 41-46.
17. Rangelova V, Gospodinov S (2003) Hybrid biosensor with biochemica amplification. Ecological Engineering and Environmental Protection 3: 44-48. 